\title{
Research Article \\ Fixed Point Theory for Admissible Type Maps with Applications
}

\author{
Ravi P. Agarwal ${ }^{1}$ and Donal O' Regan ${ }^{2}$ \\ ${ }^{1}$ Department of Mathematical Sciences, Florida Institute of Technology, Melbourne, FL 32901, USA \\ ${ }^{2}$ Department of Mathematics, National University of Ireland, Galway, Ireland
}

Correspondence should be addressed to Ravi P. Agarwal, agarwal@fit.edu

Received 8 December 2008; Accepted 18 June 2009

Recommended by Marlene Frigon

We present new Leray-Schauder alternatives, Krasnoselskii and Lefschetz fixed point theory for multivalued maps between Fréchet spaces. As an application we show that our results are directly applicable to establish the existence of integral equations over infinite intervals.

Copyright (C 2009 R. P. Agarwal and D. O'Regan. This is an open access article distributed under the Creative Commons Attribution License, which permits unrestricted use, distribution, and reproduction in any medium, provided the original work is properly cited.

\section{Introduction}

In this paper, assuming a natural sequentially compact condition we establish new fixed point theorems for Urysohn type maps between Fréchet spaces. In Section 2 we present new LeraySchauder alternatives, Krasnoselskii and Lefschetz fixed point theory for admissible type maps. The proofs rely on fixed point theory in Banach spaces and viewing a Fréchet space as the projective limit of a sequence of Banach spaces. Our theory is partly motivated by a variety of authors in the literature (see [1-6] and the references therein).

Existence in Section 2 is based on a Leray-Schauder alternative for Kakutani maps (see $[4,5,7]$ for the history of this result) which we state here for the convenience of the reader.

Theorem 1.1. Let $B$ be a Banach space, $U$ an open subset of $B$, and $0 \in U$. Suppose $T: \bar{U} \rightarrow C K(B)$ is an upper semicontinuous compact (or countably condensing) map (here $C K(B)$ denotes the family of nonempty convex compact subsets of B). Then either

(A1) $T$ has a fixed point in $\bar{U}$ or

(A2) there exists $u \in \partial U$ (the boundary of $U$ in $B$ ) and $\lambda \in(0,1)$ with $u \in \lambda T u$. 
Existence in Section 2 will also be based on the topological transversality theorem (see $[5,7]$ for the history of this result) which we now state here for the convenience of the reader. Let $B$ be a Banach space and $U$ an open subset of $B$.

Definition 1.2. We let $F \in K(\bar{U}, B)$ denote the set of all upper semicontinuous compact (or countably condensing) maps $F: \bar{U} \rightarrow C K(E)$.

Definition 1.3. We let $F \in K_{\partial U}(\bar{U}, B)$ if $F \in K(\bar{U}, B)$ with $x \notin F(x)$ for $x \in \partial U$.

Definition 1.4. A map $F \in K_{\partial U}(\bar{U}, B)$ is essential in $K_{\partial U}(\bar{U}, B)$ if for every $G \in K_{\partial U}(\bar{U}, B)$ with $\left.G\right|_{\partial U}=\left.F\right|_{\partial U}$ there exists $x \in U$ with $x \in G(x)$. Otherwise $F$ is inessential in $K_{\partial U}(\bar{U}, B)$ (i.e., there exists a fixed point free $G \in K_{\partial U}(\bar{U}, B)$ with $\left.\left.G\right|_{\partial U}=\left.F\right|_{\partial U}\right)$.

Definition 1.5. $F, G \in K_{\partial U}(\bar{U}, B)$ are homotopic in $K_{\partial U}(\bar{U}, B)$, written $F \cong G$ in $K_{\partial U}(\bar{U}, B)$, if there exists an upper semicontinuous compact (or countably condensing) map $N: \bar{U} \times$ $[0,1] \rightarrow C K(B)$ such that $N_{t}(u)=N(u, t): \bar{U} \rightarrow C K(B)$ belongs to $K_{\partial u}(\bar{U}, B)$ for each $t \in[0,1]$ and $N_{0}=F$ with $N_{1}=G$.

Theorem 1.6. Let $B$ and $U$ be as above and let $F \in K_{\partial U}(\bar{U}, B)$. Then the following conditions are equivalent:

(i) $F$ is inessential in $K_{\partial U}(\bar{U}, B)$;

(ii) there exists a map $G \in K_{\partial U}(\bar{U}, B)$ with $x \notin G(x)$ for $x \in \bar{U}$ and $F \cong G$ in $K_{\partial U}(\bar{U}, B)$.

Theorem 1.6 immediately yields the topological transversality theorem for Kakutani maps.

Theorem 1.7. Let $B$ and $U$ be as above. Suppose that $F$ and $G$ are two maps in $K_{\partial U}(\bar{U}, B)$ with $F \cong G$ in $K_{\partial U}(\bar{U}, B)$. Then $F$ is essential in $K_{\partial U}(\bar{U}, B)$ if and only if $G$ is essential in $K_{\partial U}(\bar{U}, B)$.

Also existence in Section 2 will be based on the following result of Petryshyn [8, Theorem 3].

Theorem 1.8. Let $E$ be a Banach space and let $C \subseteq E$ be a closed cone. Let $U$ and $V$ be bounded open subsets in $E$ such that $0 \in U \subseteq \bar{U} \subseteq V$ and let $F: \bar{W} \rightarrow C K(C)$ be an upper semicontinuous, $k$-set contractive (countably) map; here $0 \leq k<1, W=V \cap C$ and $\bar{W}$ denotes the closure of $W$ in $C$. Assume that

(1) $\|y\| \geq\|x\| \forall y \in F x$ and $x \in \partial \Omega$ and $\|y\| \leq\|x\| \forall y \in F x$ and $x \in \partial W$ (here $\Omega=U \cap C$ and $\partial W$ denotes the boundary of $W$ in $C$ ) or

(2) $\|y\| \leq\|x\| \forall y \in F$ and $x \in \partial \Omega$ and $\|y\| \geq\|x\| \forall y \in F x$ and $x \in \partial W$.

Then $F$ has a fixed point in $\bar{W} \backslash \Omega$.

Also in Section 2 we consider a class of maps which contain the Kakutani maps. 
Suppose that $X$ and $Y$ are Hausdorff topological spaces. Given a class $X$ of maps, $\mathcal{X}(X, Y)$ denotes the set of maps $F: X \rightarrow 2^{Y}$ (nonempty subsets of $Y$ ) belonging to $\mathcal{X}$, and $\mathcal{X}_{c}$ the set of finite compositions of maps in $\mathcal{X}$. A class $\mathcal{U}$ of maps is defined by the following properties:

(i) $\mathfrak{U}$ contains the class $\mathcal{C}$ of single-valued continuous functions;

(ii) each $F \in \boldsymbol{U}_{c}$ is upper semicontinuous and compact valued;

(iii) for any polytope $P, F \in \mathfrak{U}_{c}(P, P)$ has a fixed point, where the intermediate spaces of composites are suitably chosen for each $\mathcal{U}$.

Definition 1.9. $F \in \mathcal{U}_{c}^{\kappa}(X, Y)$ if for any compact subset $K$ of $X$, there is a $G \in \mathfrak{U}_{c}(K, Y)$ with $G(x) \subseteq F(x)$ for each $x \in K$.

The class $\mathcal{U}_{c}^{\kappa}$ is due to Park [9] and his papers include many examples in this class. Examples of $\mathcal{U}_{c}^{\kappa}$ maps are the Kakutani maps, the acyclic maps, the approximable maps, and the maps admissible in the sense of Gorniewicz.

Existence in Section 2 is based on a Leray-Schauder alternative [10] which we state here for the convenience of the reader.

Theorem 1.10. Let $E$ be a Banach space, $U$ an open convex subset of $E$, and $0 \in U$. Suppose $F \in \mathcal{U}_{c}^{\kappa}(\bar{U}, E)$ is an upper semicontinuous countably condensing map with $x \notin \lambda F x$ for $x \in \partial U$ and $\lambda \in(0,1)$. Then $F$ has a fixed point in $\bar{U}$.

Also existence in Section 2 will be based on some Lefschetz type fixed point theory. Let $X, Y$, and $\Gamma$ be Hausdorff topological spaces. A continuous single-valued map $p: \Gamma \rightarrow X$ is called a Vietoris map (written $p: \Gamma \Rightarrow X$ ) if the following two conditions are satisfied:

(i) for each $x \in X$, the set $p^{-1}(x)$ is acyclic,

(ii) $p$ is a proper map, that is, for every compact $A \subseteq X$ one has that $p^{-1}(A)$ is compact.

Let $D(X, Y)$ be the set of all pairs $X \stackrel{p}{\models} \Gamma \stackrel{q}{\rightarrow} Y$ where $p$ is a Vietoris map and $q$ is continuous. We will denote every such diagram by $(p, q)$. Given two diagrams $(p, q)$ and $\left(p^{\prime}, q^{\prime}\right)$, where $X \stackrel{p^{\prime}}{\models} \Gamma^{\prime} \stackrel{q^{\prime}}{\rightarrow} Y$, we write $(p, q) \sim\left(p^{\prime}, q^{\prime}\right)$ if there are maps $f: \Gamma \rightarrow \Gamma^{\prime}$ and $g: \Gamma^{\prime} \rightarrow \Gamma$ such that $q^{\prime} \circ f=q, p^{\prime} \circ f=p, q \circ g=q^{\prime}$, and $p \circ g=p^{\prime}$. The equivalence class of a diagram $(p, q) \in D(X, Y)$ with respect to $\sim$ is denoted by

$$
\phi=\{X \stackrel{p}{\rightleftharpoons} \Gamma \stackrel{q}{\longrightarrow} Y\}: X \longrightarrow Y
$$

or $\phi=[(p, q)]$ and is called a morphism from $X$ to $Y$. We let $M(X, Y)$ be the set of all such morphisms. For any $\phi \in M(X, Y)$ a set $\phi(x)=q p^{-1}(x)$ where $\phi=[(p, q)]$ is called an image of $x$ under a morphism $\phi$.

Consider vector spaces over a field $K$. Let $E$ be a vector space and $f: E \rightarrow E$ an endomorphism. Now let $N(f)=\left\{x \in E: f^{(n)}(x)=0\right.$ for some $\left.n\right\}$ where $f^{(n)}$ is the $n$th iterate of $f$, and let $\widetilde{E}=E \backslash N(f)$. Since $f(N(f)) \subseteq N(f)$ one has the induced endomorphism $\tilde{f}: \widetilde{E} \rightarrow \widetilde{E}$. We call $f$ admissible if $\operatorname{dim} \widetilde{E}<\infty$; for such $f$ we define the generalized trace $\operatorname{Tr}(f)$ of $f$ by putting $\operatorname{Tr}(f)=\operatorname{tr}(\tilde{f})$ where tr stands for the ordinary trace. 
Let $f=\left\{f_{q}\right\}: E \rightarrow E$ be an endomorphism of degree zero of a graded vector space $E=\left\{E_{q}\right\}$. We call $f$ a Leray endomorphism if (i) all $f_{q}$ are admissible and (ii) almost all $\widetilde{E}_{q}$ are trivial. For such $f$ we define the generalized Lefschetz number $\Lambda(f)$ by

$$
\Lambda(f)=\sum_{q}(-1)^{q} \operatorname{Tr}\left(f_{q}\right) .
$$

Let $H$ be the Čech homology functor with compact carriers and coefficients in the field of rational numbers $K$ from the category of Hausdorff topological spaces and continuous maps to the category of graded vector spaces and linear maps of degree zero. Thus $H(X)=$ $\left\{H_{q}(X)\right\}$ is a graded vector space, with $H_{q}(X)$ being the $q$-dimensional Čech homology group with compact carriers of $X$. For a continuous map $f: X \rightarrow X, H(f)$ is the induced linear map $f_{\star}=\left\{f_{\star q}\right\}$ where $f_{\star q}: H_{q}(X) \rightarrow H_{q}(X)$.

The Cech homology functor can be extended to a category of morphisms (see [11, page 364]) and also note that the homology functor $H$ extends over this category, that is, for a morphism

$$
\phi=\{X \stackrel{p}{=} \Gamma \stackrel{q}{\longrightarrow} Y\}: X \longrightarrow Y
$$

we define the induced map

$$
H(\phi)=\phi_{\star}: H(X) \longrightarrow H(Y)
$$

by putting $\phi_{\star}=q_{\star} \circ p_{\star}^{-1}$.

Let $\phi: X \rightarrow Y$ be a multivalued map (note for each $x \in X$ we assume $\phi(x)$ is a nonempty subset of $Y)$. A pair $(p, q)$ of single valued continuous maps of the form $X \stackrel{p}{\leftarrow}$ $\Gamma \stackrel{q}{\rightarrow} Y$ is called a selected pair of $\phi$ (written $(p, q) \subset \phi$ ) if the following two conditions hold:

(i) $p$ is a Vietoris map,

(ii) $q\left(p^{-1}(x)\right) \subset \phi(x)$ for any $x \in X$.

Definition 1.11. An upper semicontinuous compact map $\phi: X \rightarrow Y$ is said to be admissible (and we write $\phi \in A d(X, Y)$ ) provided that there exists a selected pair $(p, q)$ of $\phi$.

Definition 1.12. An upper semicontinuous map $\phi: X \rightarrow Y$ is said to be admissible in the sense of Gorniewicz (and we write $\phi \in A D(X, Y)$ ) provided that there exists a selected pair $(p, q)$ of $\phi$.

Definition 1.13. A map $\phi \in A d(X, X)$ is said to be a Lefschetz map if for each selected pair $(p, q) \subset \phi$ the linear map $q_{\star} p_{\star}^{-1}: H(X) \rightarrow H(X)$ (the existence of $p_{\star}^{-1}$ follows from the Vietoris theorem) is a Leray endomorphism.

If $\phi: X \rightarrow X$ is a Lefschetz map, we define the Lefschetz set $\Lambda(\phi)$ (or $\left.\Lambda_{X}(\phi)\right)$ by

$$
\Lambda(\phi)=\left\{\Lambda\left(q_{\star} p_{\star}^{-1}\right):(p, q) \subset \phi\right\}
$$


Definition 1.14. A Hausdorff topological space $X$ is said to be a Lefschetz space provided that every $\phi \in A d(X, X)$ is a Lefschetz map and $\Lambda(\phi) \neq\{0\}$ that implies $\phi$ has a fixed point.

Also we present Krasnoselskii compression and expansion theorems in Section 2 in the Fréchet space setting. Let $E=(E,|\cdot|)$ be a normed linear space and $C \subseteq E$ a closed cone. For $r>0$ let $B C(0, r)=\{x \in C:|x| \leq r\}$ and it is well known that $B C(0, r)=B(0, R) \cap C$ where $B(0, r)=\{x \in E:|x| \leq r\}$. Our next result, Theorem 1.8, was established in [12] and Theorem 1.10 can be found in [13].

Theorem 1.15. Let $E=(E,|\cdot|)$ be a normed linear space, $C \subseteq E$ a closed cone, $r, R$ constants, and $0<r<R$. Suppose that $F \in \mathcal{U}_{c}^{\kappa}(B(0, R) \cap C, C)$ is compact with

$$
\left\{\begin{array}{l}
|y| \geq|x| \quad \forall y \in F x, \quad x \in \partial B C(0, r) \\
|y| \leq|x| \quad \forall y \in F x, \quad x \in \partial B C(0, R) .
\end{array}\right.
$$

Then $F$ has a fixed point in $B C_{r, R}=\{x \in C: r \leq\|x\| \leq R\}$.

Theorem 1.16. Let $E=(E,|\cdot|)$ be a normed linear space, $C \subseteq E$ a closed cone, $r, R$ constants, and $0<r<R$. Suppose that $F \in A D(C, C)$ is completely continuous with

$$
\left\{\begin{array}{l}
|y| \leq|x| \quad \forall y \in F x, x \in \partial B C(0, r) \\
|y| \geq|x| \quad \forall y \in F x, x \in \partial B C(0, R) .
\end{array}\right.
$$

Then $F$ has a fixed point in $B C_{r, R}$.

Now let $I$ be a directed set with order $\leq$ and let $\left\{E_{\alpha}\right\}_{\alpha \in I}$ be a family of locally convex spaces. For each $\alpha \in I, \beta \in I$ for which $\alpha \leq \beta$ let $\pi_{\alpha, \beta}: E_{\beta} \rightarrow E_{\alpha}$ be a continuous map. Then the set

$$
\left\{x=\left(x_{\alpha}\right) \in \prod_{\alpha \in I} E_{\alpha}: x_{\alpha}=\pi_{\alpha, \beta}\left(x_{\beta}\right) \forall \alpha, \beta \in I, \alpha \leq \beta\right\}
$$

is a closed subset of $\prod_{\alpha \in I} E_{\alpha}$ and is called the projective limit of $\left\{E_{\alpha}\right\}_{\alpha \in I}$ and is denoted by $\lim _{\leftarrow} E_{\alpha}$ (or $\lim _{\leftarrow}\left\{E_{\alpha}, \pi_{\alpha, \beta}\right\}$ or the generalized intersection [14, page 439] $\bigcap_{\alpha \in I} E_{\alpha}$ ).

\section{Fixed Point Theory in Fréchet Spaces}

Let $E=\left(E,\left\{|\cdot|_{n}\right\}_{n \in N}\right)$ be a Fréchet space with the topology generated by a family of seminorms $\left\{|\cdot|_{n}: n \in N\right\}$; here $N=\{1,2, \ldots\}$. We assume that the family of seminorms satisfies

$$
|x|_{1} \leq|x|_{2} \leq|x|_{3} \leq \cdots \quad \text { for every } x \in E
$$

A subset $X$ of $E$ is bounded if for every $n \in N$ there exists $r_{n}>0$ such that $|x|_{n} \leq r_{n}$ for all $x \in X$. For $r>0$ and $x \in E$ we denote $B(x, r)=\left\{y \in E:|x-y|_{n} \leq r \forall n \in N\right\}$. 
To $E$ we associate a sequence of Banach spaces $\left\{\left(\mathbf{E}_{n},|\cdot|_{n}\right)\right\}$ described as follows. For every $n \in N$ we consider the equivalence relation $\sim_{n}$ defined by

$$
x \sim_{n} y \quad \text { iff }|x-y|_{n}=0 .
$$

We denote by $\mathbf{E}^{n}=\left(E / \sim_{n},|\cdot|_{n}\right)$ the quotient space, and by $\left(\mathbf{E}_{n},|\cdot|_{n}\right)$ the completion of $\mathbf{E}^{n}$ with respect to $|\cdot|_{n}$ (the norm on $\mathbf{E}^{n}$ induced by $|\cdot|_{n}$ and its extension to $\mathbf{E}_{n}$ is still denoted by $\left.|\cdot|_{n}\right)$. This construction defines a continuous map $\mu_{n}: E \rightarrow \mathbf{E}_{n}$. Now since (2.1) is satisfied the seminorm $|\cdot|_{n}$ induces a seminorm on $\mathbf{E}_{m}$ for every $m \geq n$ (again this seminorm is denoted by $|\cdot|_{n}$ ). Also (2.2) defines an equivalence relation on $\mathbf{E}_{m}$ from which we obtain a continuous map $\mu_{n, m}: \mathbf{E}_{m} \rightarrow \mathbf{E}_{n}$ since $\mathbf{E}_{m} / \sim_{n}$ can be regarded as a subset of $\mathbf{E}_{n}$. Now $\mu_{n, m} \mu_{m, k}=\mu_{n, k}$ if $n \leq m \leq k$ and $\mu_{n}=\mu_{n, m} \mu_{m}$ if $n \leq m$. We now assume the following condition holds:

$$
\left\{\begin{array}{l}
\text { for each } n \in N \text {, there exists a Banach space }\left(E_{n},|\cdot|_{n}\right) \\
\text { and an isomorphism (between normed spaces) } j_{n}: \mathbf{E}_{n} \longrightarrow E_{n}
\end{array}\right.
$$

Remark 2.1. (i) For convenience the norm on $E_{n}$ is denoted by $|\cdot|_{n}$.

(ii) In our applications $\mathbf{E}_{n}=\mathbf{E}^{n}$ for each $n \in N$.

(iii) Note if $x \in \mathbf{E}_{n}$ (or $\mathbf{E}^{n}$ ) then $x \in E$. However if $x \in E_{n}$ then $x$ is not necessaily in $E$ and in fact $E_{n}$ is easier to use in applications (even though $E_{n}$ is isomorphic to $\mathbf{E}_{n}$ ). For example if $E=C[0, \infty)$, then $\mathrm{E}^{n}$ consists of the class of functions in $E$ which coincide on the interval $[0, n]$ and $E_{n}=C[0, n]$.

Finally we assume

$$
\left\{\begin{array}{l}
E_{1} \supseteq E_{2} \supseteq \cdots \text { and for each } n \in N \\
\left|j_{n} \mu_{n, n+1} j_{n+1}^{-1} x\right|_{n} \leq|x|_{n+1} \quad \forall x \in E_{n+1}
\end{array}\right.
$$

(here we use the notation from [14], i.e., decreasing in the generalized sense) Let $\lim _{\leftarrow} E_{n}$ (or $\bigcap_{1}^{\infty} E_{n}$ where $\bigcap_{1}^{\infty}$ is the generalized intersection [14]) denote the projective limit of $\left\{E_{n}\right\}_{n \in N}$ (note $\pi_{n, m}=j_{n} \mu_{n, m} j_{m}^{-1}: E_{m} \rightarrow E_{n}$ for $m \geq n$ ) and note $\lim _{\leftarrow} E_{n} \cong E$, so for convenience we write $E=\lim _{\leftarrow} E_{n}$.

For each $X \subseteq E$ and each $n \in N$ we set $X_{n}=j_{n} \mu_{n}(X)$, and we let $\overline{X_{n}}$, int $X_{n}$ and $\partial X_{n}$ denote, respectively, the closure, the interior, and the boundary of $X_{n}$ with respect to $|\cdot|_{n}$ in $E_{n}$. Also the pseudointerior of $X$ is defined by

$$
\text { pseudo }-\operatorname{int}(X)=\left\{x \in X: j_{n} \mu_{n}(x) \in \overline{X_{n}} \backslash \partial X_{n} \text { for every } n \in N\right\}
$$

The set $X$ is pseudoopen if $X=$ pseudo $-\operatorname{int}(X)$. For $r>0$ and $x \in E_{n}$ we denote $B_{n}(x, r)=$ $\left\{y \in E_{n}:|x-y|_{n} \leq r\right\}$.

We now show how easily one can extend fixed point theory in Banach spaces to applicable fixed point theory in Fréchet spaces. In this case the map $F_{n}$ will be related to $F$ by the closure property (2.11). 
Theorem 2.2. Let $E$ and $E_{n}$ be as described above, $X$ a subset of $E$ and $F: Y \rightarrow 2^{E}$ where $\overline{\operatorname{int} X_{n}} \subseteq Y_{n}$ for each $n \in N$. Also for each $n \in N$ assume that there exists $F_{n}: \overline{\operatorname{int} X_{n}} \rightarrow 2^{E_{n}}$ and suppose the following conditions are satisfied:

$$
0 \in \text { pseudo }-\operatorname{int}(X)
$$

$$
\begin{gathered}
\left\{\begin{array}{l}
\text { for each } n \in\{2,3, \ldots\} \text { if } y \in \operatorname{int} X_{n} \text { solves } y \in F_{n} y \text { in } E_{n} \\
\text { then } j_{k} \mu_{k, n} j_{n}^{-1}(y) \in \operatorname{int} X_{k} \text { for } k \in\{1, \ldots, n-1\},
\end{array}\right. \\
\left\{\begin{array}{l}
\text { for each } n \in N, F_{n}: \overline{\operatorname{int} X_{n}} \longrightarrow C K\left(E_{n}\right) \\
\text { is an upper semicontinuous countably condensing map, }
\end{array}\right.
\end{gathered}
$$

$$
\left\{\text { for each } n \in N, y \notin \lambda F_{n} y \text { in } E_{n} \forall \lambda \in(0,1], y \in \partial \operatorname{int} X_{n}\right.
$$

$$
\left\{\begin{array}{l}
\text { for any sequence }\left\{y_{n}\right\}_{n \in N} \text { with } y_{n} \in \operatorname{int} X_{n} \\
\text { and } y_{n} \in F_{n} y_{n} \text { in } E_{n} \text { for } n \in N \text { and } \\
\text { for every } k \in N \text { there exists a subsequence } \\
N_{k} \subseteq\{k+1, k+2, \ldots\}, N_{k} \subseteq N_{k-1} \text { for } \\
k \in\{1,2, \ldots\}, N_{0}=N, \text { and a } z_{k} \in \overline{\text { int } X_{k}} \text { with } \\
j_{k} \mu_{k, n} j_{n}^{-1}\left(y_{n}\right) \longrightarrow z_{k} \text { in } E_{k} \text { as } n \longrightarrow \infty \text { in } N_{k}
\end{array}\right.
$$

$$
\left\{\begin{array}{l}
\text { if there exists } a w \in Y \text { and a sequence }\left\{y_{n}\right\}_{n \in N} \\
\text { with } y_{n} \in \operatorname{int} X_{n} \text { and } y_{n} \in F_{n} y_{n} \text { in } E_{n} \text { such that } \\
\text { for every } k \in N \text { there exists a subsequence } \\
S \subseteq\{k+1, k+2, \ldots\} \text { of } N \text { with } j_{k} \mu_{k, n} j_{n}^{-1}\left(y_{n}\right) \longrightarrow w \\
\text { in } E_{k} \text { as } n \longrightarrow \infty \text { in } S, \text { then } w \in F w \text { in } E .
\end{array}\right.
$$

Then F has a fixed point in E.

Remark 2.3. Notice that to check (2.10) we need to show that for each $k \in N$ the sequence $\left\{j_{k} \mu_{k, n} j_{n}^{-1}\left(y_{n}\right)\right\}_{n \in N_{k-1}} \subseteq \overline{\text { int } X_{k}}$ is sequentially compact. 
Proof. From Theorem 1.1 for each $n \in N$ there exists $y_{n} \in \operatorname{int} X_{n}$ with $y_{n} \in F_{n} y_{n}$ (we apply Theorem 1.1 with $U=\operatorname{int} X_{n}$ and note $j_{n} \mu_{n}(0) \in \overline{X_{n}} \backslash \partial X_{n}=$ int $X_{n}$ ). Let us look at $\left\{y_{n}\right\}_{n \in N}$. Notice $y_{1} \in \operatorname{int} X_{1}$ and $j_{1} \mu_{1, k} j_{k}^{-1}\left(y_{k}\right) \in \operatorname{int} X_{1}$ for $k \in\{2,3, \ldots\}$ from (2.7). Now (2.10) with $k=1$ guarantees that there exists a subsequence $N_{1} \subseteq\{2,3, \ldots\}$ and a $z_{1} \in \overline{\text { int } X_{1}}$ with $j_{1} \mu_{1, n} j_{n}^{-1}\left(y_{n}\right) \rightarrow z_{1}$ in $E_{1}$ as $n \rightarrow \infty$ in $N_{1}$. Look at $\left\{y_{n}\right\}_{n \in N_{1}}$. Now $j_{2} \mu_{2, n} j_{n}^{-1}\left(y_{n}\right) \in \operatorname{int} X_{2}$ for $k \in N_{1}$. Now (2.10) with $k=2$ guarantees that there exists a subsequence $N_{2} \subseteq\{3,4, \ldots\}$ of $N_{1}$ and a $z_{2} \in \overline{\operatorname{int} X_{2}}$ with $j_{2} \mu_{2, n} j_{n}^{-1}\left(y_{n}\right) \rightarrow z_{2}$ in $E_{2}$ as $n \rightarrow \infty$ in $N_{2}$. Note from (2.4) and the uniqueness of limits that $j_{1} \mu_{1,2} j_{2}^{-1} z_{2}=z_{1}$ in $E_{1}$ since $N_{2} \subseteq N_{1}$ (note $j_{1} \mu_{1, n} j_{n}^{-1}\left(y_{n}\right)=j_{1} \mu_{1,2} j_{2}^{-1} j_{2} \mu_{2, n} j_{n}^{-1}\left(y_{n}\right)$ for $n \in N_{2}$ ). Proceed inductively to obtain subsequences of integers

$$
N_{1} \supseteq N_{2} \supseteq \cdots, \quad N_{k} \subseteq\{k+1, k+2, \ldots\},
$$

and $z_{k} \in \overline{\text { int } X_{k}}$ with $j_{k} \mu_{k, n} j_{n}^{-1}\left(y_{n}\right) \rightarrow z_{k}$ in $E_{k}$ as $n \rightarrow \infty$ in $N_{k}$. Note $j_{k} \mu_{k, k+1} j_{k+1}^{-1} z_{k+1}=z_{k}$ in $E_{k}$ for $k \in\{1,2, \ldots\}$.

Fix $k \in N$. Note

$$
\begin{aligned}
z_{k} & =j_{k} \mu_{k, k+1} j_{k+1}^{-1} z_{k+1}=j_{k} \mu_{k, k+1} j_{k+1}^{-1} j_{k+1} \mu_{k+1, k+2} j_{k+2}^{-1} z_{k+2} \\
& =j_{k} \mu_{k, k+2} j_{k+2}^{-1} z_{k+2}=\cdots=j_{k} \mu_{k, m} j_{m}^{-1} z_{m}=\pi_{k, m} z_{m}
\end{aligned}
$$

for every $m \geq k$. We can do this for each $k \in N$. As a result $y=\left(z_{k}\right) \in \lim _{\leftarrow} E_{n}=E$ and also note $y \in Y$ since $z_{k} \in \overline{\operatorname{int} X_{k}} \subseteq Y_{k}$ for each $k \in N$. Also since $y_{n} \in F_{n} y_{n}$ in $E_{n}$ for $n \in N_{k}$ and $j_{k} \mu_{k, n} j_{n}^{-1}\left(y_{n}\right) \rightarrow z_{k}=y$ in $E_{k}$ as $n \rightarrow \infty$ in $N_{k}$ one has from (2.11) that $y \in F y$ in $E$.

Remark 2.4. From the proof we see that condition (2.7) can be removed from the statement of Theorem 2.2. We include it only to explain condition (2.10) (see Remark 2.3).

Remark 2.5. Note that we could replace $\overline{\operatorname{int} X_{n}} \subseteq Y_{n}$ above with $\overline{\text { int } X_{n}}$ a subset of the closure of $Y_{n}$ in $E_{n}$ if $Y$ is a closed subset of $E$ (so in this case we can take $Y=X$ if $X$ is a closed subset of $E$ ). To see this note $z_{k} \in \overline{\text { int } X_{k}}, y=\left(z_{k}\right) \in \lim _{\leftarrow} E_{n}=E$ and $\pi_{k, m}\left(y_{m}\right) \rightarrow z_{k}$ in $E_{k}$ as $m \rightarrow \infty$ and we can conclude that $y \in \bar{Y}=Y$ (note that $q \in \bar{Y}$ if and only if for every $k \in N$ there exists $\left(x_{k, m}\right) \in Y, x_{k, m}=\pi_{k, n}\left(x_{n, m}\right)$ for $n \geq k$ with $x_{k, m} \rightarrow j_{k} \mu_{k}(q)$ in $E_{k}$ as $\left.m \rightarrow \infty\right)$.

Remark 2.6. Suppose in Theorem 2.2 we replace (2.10) with

$$
\left\{\begin{array}{l}
\text { for any sequence }\left\{y_{n}\right\}_{n \in N} \text { with } y_{n} \in \text { int } X_{n} \\
\text { and } y_{n} \in F_{n} y_{n} \text { in } E_{n} \text { for } n \in N \text { and } \\
\text { for every } k \in N \text { there exists a subsequence } \\
N_{k} \subseteq\{k+1, k+2, \ldots\}, N_{k} \subseteq N_{k-1} \text { for } \\
k \in\{1,2, \ldots\}, N_{0}=N, \text { and a } z_{k} \in \text { int } X_{k} \text { with } \\
j_{k} \mu_{k, n} j_{n}^{-1}\left(y_{n}\right) \longrightarrow z_{k} \text { in } E_{k} \text { as } n \longrightarrow \infty \text { in } N_{k} .
\end{array}\right.
$$


In addition we assume $F: Y \rightarrow 2^{E}$ with $\overline{\operatorname{int} X_{n}} \subseteq Y_{n}$ for each $n \in N$ is replaced by $F: X \rightarrow 2^{E}$ and suppose (2.11) is true with $w \in Y$ replaced by $w \in X$. Then the result in Theorem 2.2 is again true.

The proof follows the reasoning in Theorem 2.2 except in this case $z_{k} \in$ int $X_{k}$ and $y \in X$.

Remark 2.7. In fact we could replace (in fact we can remove it as mentioned in Remark 2.4) (2.7) in Theorem 2.2 with

$$
\left\{\begin{array}{l}
\text { for each } n \in\{2,3, \ldots\} \text { if } y \in \text { int } X_{n} \text { solves } y \in F_{n} y \text { in } E_{n} \\
\text { then } j_{k} \mu_{k, n} j_{n}^{-1}(y) \in \overline{\operatorname{int} X_{k}} \text { for } k \in\{1, \ldots, n-1\}
\end{array}\right.
$$

and the result above is again true.

Remark 2.8. Usually in our applications one has $\partial X_{n}=\partial$ int $X_{n}$ (so $\overline{X_{n}}=\overline{\text { int } X_{n}}$ ). If $X$ is a pseudoopen subset of $E$ then for each $n \in N$ one has (see [15]) that $X_{n}$ is a open subset of $E_{n}$ so int $X_{n}=X_{n}$.

Essentially the same reasoning as in Theorem 2.2 (now using Theorem 1.7) establishes the following result. We will need the following definitions.

Let $E$ and $E_{n}$ be as described in Section 2. For the definitions below $X \subseteq E$ and $F$ : $Y \rightarrow 2^{E}$ with $\overline{\text { int } X_{n}} \subseteq Y_{n}$ for each $n \in N$ (or $\overline{\text { int } X_{n}}$ a subset of the closure of $Y_{n}$ in $E_{n}$ if $Y$ is a closed subset of $E$ ). In addition assume for each $n \in N$ that $F_{n}: \overline{\text { int } X_{n}} \rightarrow 2^{E_{n}}$.

Definition 2.9. We say $F \in K(Y, E)$ if for each $n \in N$ one has $F_{n} \in K\left(\overline{\operatorname{int} X_{n}}, E_{n}\right)$ (i.e., for each $n \in N, F_{n}: \overline{\text { int } X_{n}} \rightarrow C K\left(E_{n}\right)$ is an upper semicontinuous countably condensing map).

Definition 2.10. $F \in K_{\partial}(Y, E)$ if $F \in K(Y, E)$ and for each $n \in N$ one has $x \notin F_{n}(x)$ for $x \in$ $\partial$ int $X_{n}$.

Definition 2.11. $F \in K_{\partial}(Y, E)$ is essential in $K_{\partial}(Y, E)$ if for each $n \in N$ one has that $F_{n} \in$ $K_{\partial \text { int } X_{n}}\left(\overline{\text { int } X_{n}}, E_{n}\right)$ is essential in $K_{\partial}$ int $X_{n}\left(\overline{\text { int } X_{n}}, E_{n}\right)$ (i.e., for each $n \in N$, every map $G_{n} \in$ $K_{\partial}$ int $X_{n}\left(\overline{\text { int } X_{n}}, E_{n}\right)$ with $\left.G_{n}\right|_{\partial}$ int $X_{n}=\left.F_{n}\right|_{\partial}$ int $X_{n}$ has a fixed point in int $\left.X_{n}\right)$.

Remark 2.12. Note that if $j_{n} \mu_{n}(0) \in U_{n}$ for each $n \in N$ then $0 \in K_{\partial}(Y, E)$ is essential in $K_{\partial}(Y, E)$ (see [7]).

Definition 2.13. (We assume $j_{n} \mu_{n}(0) \in$ int $X_{n}$ for $n \in N$.) $F, 0 \in K_{\partial}(Y, E)$ are homotopic in $K_{\partial}(Y, E)$, written $F \cong G$ in $K_{\partial}(Y, E)$, if for each $n \in N$ one has $F_{n} \cong j_{n} \mu_{n}(0)$ in $K_{\partial}$ int $X_{n}\left(\overline{\text { int } X_{n}}, E_{n}\right)$.

Theorem 2.14. Let $E$ and $E_{n}$ be as described above, $X$ a subset of $E$ and $F: Y \rightarrow 2^{E}$ where $\overline{\text { int }} X_{n} \subseteq$ $Y_{n}$ for each $n \in N$ or int $X_{n}$ a subset of the closure of $Y_{n}$ in $E_{n}$ (if $Y$ is a closed subset of $E$ ). Also for 
each $n \in N$ assume that there exists $F_{n}: \overline{\text { int } X_{n}} \rightarrow 2^{E_{n}}$ and suppose $F \in K_{\partial}(Y, E),(2.6),(2.7)$, and the following condition holds:

$$
F \cong 0 \text { in } K_{\partial}(Y, E)
$$

Also assume (2.10) and (2.11) hold. Then $F$ has a fixed point in $E$.

Proof. Fix $n \in N$. Now Remark 2.12 guarantees that the zero map (i.e., $G(x)=j_{n} \mu_{n}(0)$ ) is essential in $K_{\partial U_{n}}\left(\overline{U_{n}}, E_{n}\right)$ for each $n \in N$. Now Theorem 1.7 guarantees that $F_{n}$ is essential in $K_{\partial U_{n}}\left(\overline{U_{n}}, E_{n}\right)$ so in particular there exists $y_{n} \in U_{n}$ with $y_{n} \in F_{n} y_{n}$. Essentially the same reasoning as in Theorem 2.2 (with Remark 2.5) establishes the result.

Remark 2.15. Notice that (2.6) and (2.17) could be replaced by $F \cong G$ in $K_{\partial}(Y, E)$ (of course we assume $G \in K_{\partial}(Y, E)$ and we must specify $G_{n}$ for $n \in N$ here).

Remark 2.16. Condition (2.7) can be removed from the statement of Theorem 2.14.

Remark 2.17. Note that Remark 2.6 holds in this situation also.

As an application of Theorem 2.2 we discuss the integral equation

$$
y(t)=\int_{0}^{\infty} K(t, s) f(s, y(s)) d s \quad \text { for } t \in[0, \infty)
$$

Theorem 2.18. Let $1 \leq p<\infty$ be a constant and $1<q \leq \infty$ the conjugate to $p$. Suppose the following conditions are satisfied:

for each $t \in[0, \infty)$, the map $s \longmapsto K(t, s)$ is measurable,

$$
\sup _{t \in[0, \infty)}\left(\int_{0}^{\infty}|K(t, s)|^{q} d s\right)^{1 / q}<\infty
$$

$\int_{0}^{\infty}\left|K\left(t^{\prime}, s\right)-K(t, s)\right|^{q} d s \longrightarrow 0$ as $t \longrightarrow t^{\prime}$, for each $t^{\prime} \in[0, \infty)$,

$\left(f:[0, \infty) \times \mathbf{R} \rightarrow \mathbf{R}\right.$ is a $L^{p}$-Carathéodory function:

by this one means

(a) the map $t \longmapsto f(t, y)$ is measurable for all $y \in \mathbf{R}$;

(b) the map $y \longmapsto f(t, y)$ is continuous for a.e. $t \in[0, \infty)$;

(c) for each $r>0$ there exists $\mu_{r} \in L^{p}[0, \infty)$ such that $|y| \leq r$ implies $|f(t, y)| \leq \mu_{r}(t)$ for a.e. $t \in[0, \infty)$, 
Fixed Point Theory and Applications

$$
\begin{aligned}
& \left\{\begin{array}{l}
\text { there exists a continuous nondecreasing function } \\
\psi:[0, \infty) \longrightarrow[0, \infty) \text { and } a \phi \in L^{p}[0, \infty) \text { with } \\
|f(s, y)| \leq \phi(s) \psi(|y|) \forall y \in \mathbf{R} \\
\text { and a.e. } s \in[0, \infty),
\end{array}\right. \\
& \exists r>0 \text { with } r>K_{1} \psi(r) \text { where } K_{1}=\sup _{t \in[0, \infty)} \int_{0}^{\infty} \phi(s)|K(t, s)| d s .
\end{aligned}
$$

Then (2.17) has at least one solution in $C[0, \infty)$.

Remark 2.19. One could also obtain a multivalued version of Theorem 2.18 by using the ideas in the proof below with the ideas in [16].

Proof. Here $E=C[0, \infty), E^{k}$ consists of the class of functions in $E$ which coincide on the interval $[0, k], E_{k}=C[0, k]$ with of course $\pi_{n, m}=j_{n} \mu_{n, m} j_{m}^{-1}: E_{m} \rightarrow E_{n}$ defined by $\pi_{n, m}(x)=\left.x\right|_{[0, n]}$. We will apply Theorem 2.2 with

$$
X=\left\{u \in C[0, \infty):|u|_{n} \leq r \text { for each } n \in N\right\},
$$

here $|u|_{n}=\sup _{t \in[0, n]}|u(t)|$. Fix $n \in N$ and note

$$
X_{n}=\overline{X_{n}}=\left\{u \in C[0, n]:|u|_{n} \leq r\right\}
$$

with

$$
\text { int } X_{n}=\left\{u \in C[0, n]:|u|_{n}<r\right\} \text {. }
$$

Let $F_{n}: \overline{\text { int } X_{n}} \rightarrow E_{n}$ be given by

$$
F_{n} y(t)=\int_{0}^{n} K(t, s) f(s, y(s)) d s
$$

Also let $Y=X$ (we will use Remark 2.5) and let $F: Y \rightarrow E$ be given by

$$
F y(t)=\int_{0}^{\infty} K(t, s) f(s, y(s)) d s
$$

Clearly (2.6) and (2.7) hold, and a standard argument in the literature guarantees that $F$ : $\overline{\text { int } X_{n}} \rightarrow E_{n}$ is continuous and compact so (2.8) holds. To show (2.9) fix $n \in N$ and suppose that there exists $x \in \partial$ int $X_{n}\left(\right.$ so $\left.|x|_{n}=r\right)$ and $\lambda \in(0,1]$ with $x=\lambda F_{n} x$. Then for $t \in[0, n]$ one has

$$
|x(t)| \leq \psi\left(|x|_{n}\right) \int_{0}^{n}|K(t, s)| \phi(s) d s \leq \psi\left(|x|_{n}\right) K_{1},
$$


so $|x|_{n} \leq \psi\left(|x|_{n}\right) K_{1}$, that is, $r \leq \psi(r) K_{1}$. This contradicts (2.23), so (2.9) holds. To show (2.10) consider a sequence $\left\{y_{n}\right\}_{n \in N}$ with $y_{n} \in C[0, n], y_{n}=F_{n} y_{n}$ on $[0, n]$ and $\left|y_{n}\right|_{n}<r$. Now to show (2.10) we will show for a fixed $k \in N$ that $\left\{j_{k} \mu_{k, n} j_{n}^{-1}\left(y_{n}\right)\right\}_{n \in S} \subseteq \overline{\text { int } X_{k}}$ is sequentially compact for any subsequence $S$ of $\{k, k+1, \ldots\}$. Note for $n \in S$ that $j_{k} \mu_{k, n} j_{n}^{-1}\left(y_{n}\right)=\left.y_{n}\right|_{[0, k]}$ so $\left\{j_{k} \mu_{k, n} j_{n}^{-1}\left(y_{n}\right)\right\}_{n \in S}$ is uniformly bounded since $\left|y_{n}\right|_{n} \leq r$ for $n \in S$ implies $\left|y_{n}\right|_{k} \leq r$ for $n \in S$. Also $\left\{j_{k} \mu_{k, n} j_{n}^{-1}\left(y_{n}\right)\right\}_{n \in S}$ is equicontinuous on $[0, k]$ since for $n \in S$ and $t, x \in[0, k]$ (note there exists $h_{r} \in L^{p}[0, \infty)$ with $\left|f\left(s, y_{n}(s)\right)\right| \leq h_{\mathrm{r}}(s)$ for a.e. $\left.s \in[0, n]\right)$ one has

$$
\begin{aligned}
& \left|j_{k} \mu_{k, n} j_{n}^{-1}\left(y_{n}(t)\right)-j_{k} \mu_{k, n} j_{n}^{-1}\left(y_{n}(x)\right)\right| \\
& \quad \leq \int_{0}^{n}|K(t, s)-K(x, s)|\left|f\left(s, y_{n}(s)\right)\right| d s \\
& \quad \leq\left(\int_{0}^{\infty}\left[h_{r}(s)\right]^{p} d s\right)^{1 / p}\left(\int_{0}^{\infty}|K(t, s)-K(x, s)|^{q} d s\right)^{1 / q} .
\end{aligned}
$$

The Arzela-Ascoli theorem guarantees that $\left\{j_{k} \mu_{k, n} j_{n}^{-1}\left(y_{n}\right)\right\}_{n \in S} \subseteq \overline{\text { int } X_{k}}$ is sequentially compact. Finally we show (2.11). Suppose there exists $w \in C[0, \infty)$ and a sequence $\left\{y_{n}\right\}_{n \in N}$ with $y_{n} \in$ int $X_{n}$ and $y_{n}=F_{n} y_{n}$ in $C[0, n]$ such that for every $k \in N$ there exists a subsequence $S \subseteq\{k+1, k+2, \ldots\}$ of $N$ with $y_{n} \rightarrow w$ in $C[0, k]$ as $n \rightarrow \infty$ in $S$. If we show

$$
w(t)=\int_{0}^{\infty} K(t, s) f(s, w(s)) d s \quad \text { for } t \in[0, \infty)
$$

then (2.11) holds. To see (2.31) fix $t \in[0, \infty)$. Consider $k \geq t$ and $n \in S$ (as described above). Then $y_{n}=F_{n} y_{n}$ for $n \in S$ and so

$$
y_{n}(t)-\int_{0}^{k} K(t, s) f\left(s, y_{n}(s)\right) d s=\int_{k}^{n} K(t, s) f\left(s, y_{n}(s)\right) d s,
$$

so

$$
\left|j_{k} \mu_{k, n} j_{n}^{-1}\left(y_{n}(t)\right)-\int_{0}^{k} K(t, s) f\left(s, j_{k} \mu_{k, n} j_{n}^{-1}\left(y_{n}(s)\right)\right) d s\right| \leq \int_{k}^{n}|K(t, s)| h_{r}(s) d s .
$$

(here (2.21) guarantees that there exists $h_{r} \in L^{p}[0, \infty)$ with $\left|f\left(s, y_{n}(s)\right)\right| \leq h_{r}(s)$ for a.e. $s \in[0, n])$ Let $n \rightarrow \infty$ through $S$ and use the Lebesgue Dominated Convergence theorem to obtain

$$
\left|w(t)-\int_{0}^{k} K(t, s) f(s, w(s)) d s\right| \leq \int_{k}^{\infty}|K(t, s)| h_{r}(s) d s
$$


since $j_{k} \mu_{k, n} j_{n}^{-1}\left(y_{n}\right) \rightarrow w$ in $C[0, k]$. Finally let $k \rightarrow \infty$ (note (2.19)) to obtain

$$
w(t)-\int_{0}^{\infty} K(t, s) f(s, w(s)) d s=0
$$

Thus (2.11) holds. Our result now follows from Theorem 2.2 (with Remark 2.5).

Essentially the same reasoning as in Theorem 2.2 (now using Theorem 1.8) establishes the following result.

Theorem 2.20. Let $E$ and $E_{n}$ be as described in the beginning of Section 2, $C$ a closed cone in $E, U$, and $V$ are bounded pseudoopen subsets of $E$ with $0 \in U \subseteq \bar{U} \subseteq V$, and $F: Y \rightarrow 2^{E}$. Also assume either $\overline{W_{n}}=\overline{V_{n} \cap \overline{C_{n}}} \subseteq Y_{n}$ for each $n \in N$ (here $W_{n}=V_{n} \cap \overline{C_{n}}$ ) or $\overline{V_{n} \cap \overline{C_{n}}}$ a subset of the closure of $Y_{n}$ in $E_{n}$ (if $Y$ is a closed subset of $E$ ). Also for each $n \in N$ assume $F_{n}: \overline{W_{n}} \rightarrow 2^{E_{n}}$ and suppose that the following conditions hold (here $\Omega_{n}=\overline{C_{n}} \cap U_{n}$ ):

$$
\left\{\begin{array}{l}
\text { for each } n \in\{2,3, \ldots\} \text { if } y \in \overline{W_{n}} \backslash \Omega_{n} \text { solves } y \in F_{n} y \\
\text { in } E_{n} \text { then } j_{k} \mu_{k, n} j_{n}^{-1}(y) \in \overline{W_{k}} \text { for } k \in\{1, \ldots, n-1\},
\end{array}\right.
$$

$$
\left\{\begin{array}{l}
\text { for each } n \in N, F_{n}: \overline{W_{n}} \longrightarrow C K\left(\overline{C_{n}}\right) \text { is an upper } \\
\text { semicontinuous } k \text {-set (countably) contractive map } \\
\text { (here }(0 \leq k<1)) \text {. }
\end{array}\right.
$$

Also for each $n \in N$ assume either

$$
\left\{\begin{array}{l}
|y|_{n} \geq|x|_{n} \quad \forall y \in F_{n} x, \forall x \in \partial \Omega_{n} \\
|y|_{n} \leq|x|_{n} \quad \forall y \in F_{n} x, \quad \forall x \in \partial W_{n}
\end{array}\right.
$$

or

$$
\begin{cases}|y|_{n} \leq|x|_{n} \quad \forall y \in F_{n} x, \forall x \in \partial \Omega_{n} \\ |y|_{n} \geq|x|_{n} \quad \forall y \in F_{n} x, \forall x \in \partial W_{n}\end{cases}
$$

hold. Finally suppose that the following hold:

$$
\left\{\begin{array}{l}
\text { for every } k \in N \text { and any subsequence } A \subseteq\{k, k+1, \ldots\} \\
\text { if } x \in \overline{C_{n}} \text { is such that } x \in \overline{W_{n}} \backslash \Omega_{n} \text { for some } n \in A \\
\text { then there exists a } \gamma>0 \text { with }\left|j_{k} \mu_{k, n} j_{n}^{-1} x\right|_{k} \geq \gamma,
\end{array}\right.
$$




$$
\left\{\begin{array}{l}
\text { for any sequence }\left\{y_{n}\right\}_{n \in N} \text { with } y_{n} \in \overline{W_{n}} \backslash \Omega_{n} \\
\text { and } y_{n} \in F_{n} y_{n} \text { in } E_{n} \text { for } n \in N \text { and } \\
\text { for every } k \in N \text { there exists a subsequence } \\
N_{k} \subseteq\{k+1, k+2, \ldots\}, N_{k} \subseteq N_{k-1} \text { for } \\
k \in\{1,2, \ldots\}, N_{0}=N, \text { and a } z_{k} \in \overline{W_{k}} \text { with } \\
j_{k} \mu_{k, n} j_{n}^{-1}\left(y_{n}\right) \longrightarrow z_{k} \text { in } E_{k} \text { as } n \longrightarrow \infty \text { in } N_{k},
\end{array}\right.
$$

$$
\left\{\begin{array}{l}
\text { if there exists } a w \in Y \text { and a sequence }\left\{y_{n}\right\}_{n \in N} \\
\text { with } y_{n} \in \overline{W_{n}} \backslash \Omega_{n} \text { and } y_{n} \in F_{n} y_{n} \text { in } E_{n} \text { such that } \\
\text { for every } k \in N \text { there exists a subsequence } \\
S \subseteq\{k+1, k+2, \ldots\} \text { of } N \text { with } j_{k} \mu_{k, n} j_{n}^{-1}\left(y_{n}\right) \longrightarrow w \\
\text { in } E_{k} \text { as } n \longrightarrow \infty \text { in } S, \text { then } w \in F w \text { in } E .
\end{array}\right.
$$

Then $F$ has a fixed point in $E$.

Proof. Fix $n \in N$. We would like to apply Theorem 1.8. Note that we know from [15] that $\overline{C_{n}}$ is a cone and $U_{n}$ and $V_{n}$ are open and bounded with $j_{n} \mu_{n}(0) \in U_{n} \subseteq \overline{U_{n}} \subseteq V_{n}$. Theorem 1.8 guarantees that there exists $y_{n} \in \overline{W_{n}} \backslash \Omega_{n}$ with $y_{n} \in F_{n} y_{n}$ in $E_{n}$. As in Theorem 2.2 there exists a subsequence $N_{1} \subseteq\{2,3, \ldots\}$ and a $z_{1} \in \overline{W_{1}}$ with $j_{1} \mu_{1, n} j_{n}^{-1}\left(y_{n}\right) \rightarrow z_{1}$ in $E_{1}$ as $n \rightarrow \infty$ in $N_{1}$. Also $y_{n} \in \overline{W_{n}} \backslash \Omega_{n}$ together with (2.40) yields $\left|j_{1} \mu_{1, n} j_{n}^{-1}\left(y_{n}\right)\right|_{1} \geq \gamma$ for $n \in N$ and so $\left|z_{1}\right|_{1} \geq \gamma$. Proceed inductively to obtain subsequences of integers

$$
N_{1} \supseteq N_{2} \supseteq \cdots, \quad N_{k} \subseteq\{k+1, k+2, \ldots\}
$$

and $z_{k} \in \overline{W_{k}}$ with $j_{k} \mu_{k, n} j_{n}^{-1}\left(y_{n}\right) \rightarrow z_{k}$ in $E_{k}$ as $n \rightarrow \infty$ in $N_{k}$. Note $j_{k} \mu_{k, k+1} j_{k+1}^{-1} z_{k+1}=$ $z_{k}$ in $E_{k}$ for $k \in\{1,2, \ldots\}$ and $\left|z_{k}\right|_{k} \geq \gamma$. Now essentially the same reasoning as in Theorem 2.2 (with Remark 2.5) guarantees the result.

Remark 2.21. Condition (2.36) can be removed from the statement of Theorem 2.20.

Remark 2.22. Note (2.40) is only needed to guarantee that the fixed point $y$ satisfies $\left|j_{k} \mu_{k}(y)\right|_{k} \geq \gamma$ for $k \in N$. If we assume all the conditions in Theorem 2.20 except (2.40) then again $F$ has a fixed point in $E$ but the above property is not guaranteed.

Essentially the same reasoning as in Theorem 2.2 (just apply Theorem 1.10 in this case) establishes the following result.

Theorem 2.23. Let $E$ and $E_{n}$ be as described above, $X$ a convex subset of $E$, and $F: Y \rightarrow 2^{E}$ where $\overline{\text { int } X_{n}} \subseteq Y_{n}$ for each $n \in N$ or int $X_{n}$ a subset of the closure of $Y_{n}$ in $E_{n}$ (if $Y$ is a closed subset of $E$ ). 
Also for each $n \in N$ assume that there exists $F_{n}: \overline{\text { int } X_{n}} \rightarrow 2^{E_{n}}$ and suppose that (2.6), (2.7), (2.9), (2.10), (2.11) and the following condition hold:

$$
\left\{\begin{array}{l}
\text { for each } n \in N, F_{n} \in \mathcal{U}_{c}^{\kappa}\left(\overline{\operatorname{int} X_{n}}, E_{n}\right) \text { is an } \\
\text { upper semicontinuous countably condensing map. }
\end{array}\right.
$$

Then F has a fixed point in $E$.

Proof. Fix $n \in N$. We would like to apply Theorem 1.10. Note that we know from [15] that int $X_{n}$ is convex. From Theorem 1.10 for each $n \in N$ there exists $y_{n} \in$ int $X_{n}$ with $y_{n} \in F_{n} y_{n}$ in $E_{n}$. Now essentially the same reasoning as in Theorem 2.2 (with Remark 2.5) guarantees the result.

Remark 2.24. Note Remarks 2.4, 2.6, and 2.7 hold in this situation also.

Now we present some Lefschetz type theorems in Fréchet spaces. Let $E$ and $E_{n}$ be as described above.

Definition 2.25. A set $A \subseteq E$ is said to be PRLS if for each $n \in N, A_{n} \equiv j_{n} \mu_{n}(A)$ is a Lefschetz space.

Definition 2.26. A set $A \subseteq E$ is said to be CPRLS if for each $n \in N, \overline{A_{n}}$ is a Lefschetz space.

Theorem 2.27. Let $E$ and $E_{n}$ be as described above, $C \subseteq E$ is an PRLS, and $F: C \rightarrow 2^{E}$. Also for each $n \in N$ assume that there exists $F_{n}: C_{n} \rightarrow 2^{E_{n}}$ and suppose that the following conditions are satisfied:

$$
\left\{\begin{array}{l}
\text { for each } n \in\{2,3, \ldots\} \text { if } y \in C_{n} \text { solves } y \in F_{n} y \text { in } E_{n} \\
\text { then } j_{k} \mu_{k, n} j_{n}^{-1}(y) \in C_{k} \text { for } k \in\{1, \ldots, n-1\}
\end{array}\right.
$$

$$
\text { for each } n \in N, \quad F_{n} \in A d\left(C_{n}, C_{n}\right) \text {, }
$$

$$
\text { for each } n \in N, \quad \Lambda_{C_{n}}\left(F_{n}\right) \neq\{0\},
$$

$$
\left\{\begin{array}{l}
\text { for any sequence }\left\{y_{n}\right\}_{n \in N} \text { with } y_{n} \in C_{n} \\
\text { and } y_{n} \in F_{n} y_{n} \text { in } E_{n} \text { for } n \in N \text { and } \\
\text { for ever } y \in N \text { there exists a subsequence } \\
N_{k} \subseteq\{k+1, k+2, \ldots\}, N_{k} \subseteq N_{k-1} \text { for } \\
k \in\{1,2, \ldots\}, N_{0}=N, \text { and a } z_{k} \in C_{k} \text { with } \\
j_{k} \mu_{k, n} j_{n}^{-1}\left(y_{n}\right) \longrightarrow z_{k} \text { in } E_{k} \text { as } n \longrightarrow \infty \text { in } N_{k},
\end{array}\right.
$$




$$
\left\{\begin{array}{l}
\text { if there exists a } w \in C \text { and a sequence }\left\{y_{n}\right\}_{n \in N} \\
\text { with } y_{n} \in C_{n} \text { and } y_{n} \in F_{n} y_{n} \text { in } E_{n} \text { such that } \\
\text { for every } k \in N \text { there exists a subsequence } \\
S \subseteq\{k+1, k+2, \ldots\} \text { of } N \text { with } j_{k} \mu_{k, n} j_{n}^{-1}\left(y_{n}\right) \longrightarrow w \\
\text { in } E_{k} \text { as } n \longrightarrow \infty \text { in } S, \text { then } w \in F w \text { in } E .
\end{array}\right.
$$

Then $F$ has a fixed point in $E$.

Proof. For each $n \in N$ there exists $y_{n} \in C_{n}$. Now the same reasoning as in Theorem 2.2 guarantees the result.

Remark 2.28. Condition (2.45) can be removed from the statement of Theorem 2.27.

Remark 2.29. Suppose in Theorem 2.27, one has

$$
\left\{\begin{array}{l}
\text { for any sequence }\left\{y_{n}\right\}_{n \in N} \text { with } y_{n} \in C_{n} \\
\text { and } y_{n} \in F_{n} y_{n} \text { in } E_{n} \text { for } n \in N \text { and } \\
\text { for every } k \in N \text { there exists a subsequence } \\
N_{k} \subseteq\{k+1, k+2, \ldots\}, N_{k} \subseteq N_{k-1} \text { for } \\
k \in\{1,2, \ldots\}, N_{0}=N, \text { and a } z_{k} \in \overline{C_{k}} \text { with } \\
j_{k} \mu_{k, n} j_{n}^{-1}\left(y_{n}\right) \longrightarrow z_{k} \text { in } E_{k} \text { as } n \longrightarrow \infty \text { in } N_{k}
\end{array}\right.
$$

instead of (2.48) and $F: C \rightarrow 2^{E}$ is replaced by $F: Y \rightarrow 2^{E}$ with $C \subseteq Y$ and $\overline{C_{n}} \subseteq Y_{n}$ for each $n \in N$ and suppose that (2.49) is true with $w \in C$ replaced by $w \in Y$. Then the result in Theorem 2.27 is again true.

In fact we could replace $\overline{C_{n}} \subseteq Y_{n}$ above with $\overline{C_{n}}$ a subset of the closure of $Y_{n}$ in $E_{n}$ if $Y$ is a closed subset of $E$ (so in this case we can take $Y=C$ if $C$ is a closed subset of $E$ ).

In fact in this remark we could replace (in fact we can remove it as mentioned in Remark 2.4) (2.45) with

$$
\left\{\begin{array}{l}
\text { for each } n \in\{2,3, \ldots\} \text { if } y \in C_{n} \text { solves } y \in F_{n} y \text { in } E_{n} \\
\text { then } j_{k} \mu_{k, n} j_{n}^{-1}(y) \in \overline{C_{k}} \text { for } k \in\{1, \ldots, n-1\}
\end{array}\right.
$$

and the result above is again true. 
Also one has the following result.

Theorem 2.30. Let $E$ and $E_{n}$ be as described above, $C \subseteq E$ is an CPRLS and $F: C \rightarrow 2^{E}$. Also assume $C$ is a closed subset of $E$ and for each $n \in N$ that $F_{n}: \overline{C_{n}} \rightarrow 2^{E_{n}}$ and suppose that the following conditions are satisfied:

$$
\left\{\begin{array}{l}
\text { for each } n \in\{2,3, \ldots\} \text { if } y \in \overline{C_{n}} \text { solves } y \in F_{n} y \text { in } E_{n} \\
\text { then } j_{k} \mu_{k, n} j_{n}^{-1}(y) \in \overline{C_{k}} \text { for } k \in\{1, \ldots, n-1\}
\end{array}\right.
$$

$$
\text { for each } n \in N, \quad F_{n} \in \operatorname{Ad}\left(\overline{C_{n}}, \overline{C_{n}}\right) \text {, }
$$

$$
\text { for each } n \in N, \quad \Lambda_{\overline{C_{n}}}\left(F_{n}\right) \neq\{0\} \text {, }
$$

$$
\left\{\begin{array}{l}
\text { for any sequence }\left\{y_{n}\right\}_{n \in N} \text { with } y_{n} \in \overline{C_{n}} \\
\text { and } y_{n} \in F_{n} y_{n} \text { in } E_{n} \text { for } n \in N \text { and } \\
\text { for every } k \in N \text { there exists a subsequence } \\
N_{k} \subseteq\{k+1, k+2, \ldots\}, N_{k} \subseteq N_{k-1} \text { for } \\
k \in\{1,2, \ldots\}, N_{0}=N, \text { and a } z_{k} \in \overline{C_{k}} \text { with } \\
j_{k} \mu_{k, n} j_{n}^{-1}\left(y_{n}\right) \longrightarrow z_{k} \text { in } E_{k} \text { as } n \longrightarrow \infty \text { in } N_{k},
\end{array}\right.
$$

$$
\left\{\begin{array}{l}
\text { if there exists a } w \in C \text { and a sequence }\left\{y_{n}\right\}_{n \in N} \\
\text { with } y_{n} \in \overline{C_{n}} \text { and } y_{n} \in F_{n} y_{n} \text { in } E_{n} \text { such that } \\
\text { for every } k \in N \text { there exists a subsequence } \\
S \subseteq\{k+1, k+2, \ldots\} \text { of } N \text { with } j_{k} \mu_{k, n} j_{n}^{-1}\left(y_{n}\right) \longrightarrow w \\
\text { in } E_{k} \text { as } n \longrightarrow \infty \text { in } S, \text { then } w \in F w \text { in } E .
\end{array}\right.
$$

Then $F$ has a fixed point in $E$.

Remark 2.31. Condition (2.52) can be removed from the statement of Theorem 2.30.

Remark 2.32. Note that we can remove the assumption in Theorem 2.30 that $C$ is a closed subset of $E$ if we assume $F: Y \rightarrow 2^{E}$ with $C \subseteq Y$ and $\overline{C_{n}} \subseteq Y_{n}$ (or $\overline{C_{n}}$ a subset of the closure of $Y_{n}$ in $E_{n}$ if $Y$ is a closed subset of $E$ ) for each $n \in N$ with of course $w \in C$ replaced by $w \in Y$ in (2.56). 
Remark 2.33. Of course there are analogue results for compact morphisms (see the ideas here and in [17]) and for compact permissible maps (see [18]).

Next we present some Krasnoselskii results in the Fréchet space setting (in the first we use Theorem 1.15 and the second Theorem 1.16).

Theorem 2.34. Let $E$ and $E_{n}$ be as described in the beginning of Section 2, $C$ a closed cone in $E, r$, and $R$ are constants with $0<r<R$, and $F: Y \rightarrow 2^{E}$ with $Y \subseteq E$, and $\overline{W_{n}}=B_{n}(0, R) \cap \overline{C_{n}} \subseteq Y_{n}$ (or $\overline{W_{n}}$ is a subset of the closure of $Y_{n}$ in $E_{n}$ if $Y$ is a closed subset of $E$ ) for each $n \in N$; here $W_{n}=\overline{C_{n}} \cap V_{n}$ where $V_{n}=\left\{x \in E_{n}:|x|_{n}<R\right\}$. Also for each $n \in N$ assume $F_{n}: \overline{W_{n}} \rightarrow 2^{E_{n}}$ and suppose that (2.36) and the following conditions are satisfied (here $\Omega_{n}=U_{n} \cap \overline{C_{n}}$ with $U_{n}=\left\{x \in E_{n}:|x|_{n}<r\right\}$ ):

$$
\left\{\begin{array}{l}
\text { for each } n \in N, \quad F_{n} \in \mathcal{U}_{c}^{\kappa}\left(B_{n}(0, R) \cap \overline{C_{n}}, \overline{C_{n}}\right) \\
\text { is an upper semicontinuous compact map, }
\end{array}\right.
$$

$$
\left\{\begin{array}{l}
|y|_{n} \geq|x|_{n} \quad \forall y \in F_{n} x, x \in \partial \Omega_{n} \\
|y|_{n} \leq|x|_{n} \quad \forall y \in F_{n} x, \quad x \in \partial W_{n} .
\end{array}\right.
$$

Also assume (2.40), (2.41), and (2.42) hold. Then $F$ has a fixed point in E.

Remark 2.35. Note Remarks 2.21 and 2.22 hold in this situation also.

Theorem 2.36. Let $E$ and $E_{n}$ be as described in the beginning of Section 2, $C$ a closed cone in $E$, $r$, and $R$ are constants with $0<r<R$, and $F: Y \rightarrow 2^{E}$ with $Y \subseteq E, C \subseteq Y$ and $\overline{C_{n}} \subseteq Y_{n}$ (or $\overline{C_{n}}$ is a subset of the closure of $Y_{n}$ in $E_{n}$ if $Y$ is a closed subset of E) for each $n \in N$. Also for each $n \in N$ assume $F_{n}: \overline{C_{n}} \rightarrow 2^{E_{n}}$ and suppose that (2.36) and the following conditions are satisfied (here $W_{n}=\overline{C_{n}} \cap V_{n}$ where $V_{n}=\left\{x \in E_{n}:|x|_{n}<R\right\}$ and $\Omega_{n}=U_{n} \cap \overline{C_{n}}$ with $\left.U_{n}=\left\{x \in E_{n}:|x|_{n}<r\right\}\right)$ :

$$
\text { for each } n \in N, \quad F_{n} \in A D\left(\overline{C_{n}}, \overline{C_{n}}\right) \text { is a completely continuous map, }
$$

$$
\left\{\begin{array}{l}
|y|_{n} \leq|x|_{n} \quad \forall y \in F_{n} x, x \in \partial \Omega_{n} \\
|y|_{n} \geq|x|_{n} \quad \forall y \in F_{n} x, \quad x \in \partial W_{n} .
\end{array}\right.
$$

In addition assume (2.40), (2.41), and (2.42) hold. Then F has a fixed point in E.

To conclude the paper we apply Theorem 2.20 (or Theorem 2.36) to (2.17).

Theorem 2.37. Let $1 \leq p \leq \infty$ be a constant and $q$ the conjugate to $p$ and suppose that (2.18), (2.19), (2.20), (2.21), (2.22), and (2.23) hold. In addition assume the following conditions are satisfied: 
Fixed Point Theory and Applications

$$
\begin{aligned}
& \left\{\begin{array}{l}
\text { for each } t \in[0, \infty) \text { one has } k(t, s) \geq 0 \text { for a.e. } s \in[0, \infty), \\
\text { and } f:[0, \infty) \times \mathbf{R} \rightarrow[0, \infty) \text { with } f(s, u)>0 \text { for }(s, u) \in[0, \infty) \times(0, \infty),
\end{array}\right. \\
& \left\{\begin{array}{l}
\text { there exists } g:[0, \infty) \longrightarrow(0, \infty) \text { with } g \in L^{q}[0, \infty) \\
\text { and with } k(t, s) \leq g(s) \text { for } t \in[0, \infty),
\end{array}\right. \\
& \left\{\begin{array}{l}
\text { there exists } a, b \in[0,1], \quad a<b, \text { and } M, \quad 0<M<1 \\
\text { with } k(t, s) \geq M g(s) \text { for } t \in[a, b] \text { and a.e. } s \in[0, \infty),
\end{array}\right. \\
& \left\{\begin{array}{l}
\text { there exists } \tau \in L^{p}[a, b] \text { with } f(s, u) \geq \tau(s) \psi(u) \\
\text { for a.e. } s \in[a, b] \text { and all } u \in[0, \infty),
\end{array}\right. \\
& \exists R>r \text { with } R<\psi(M R) \sup _{t \in[0,1]} \int_{a}^{b} \tau(s) k(t, s) d s .
\end{aligned}
$$

Then (2.17) has at least one solution in $y \in C[0, \infty)$ with $M r \leq|y|_{n} \leq R$ for $n \in N$.

Remark 2.38. One could obtain a multivalued version of Theorem 2.37 by using the ideas in the proof below with the ideas in [16].

Remark 2.39. In (2.63) we picked $b \in[0,1]$ for convenience (i.e., so we could take $n \in N$; otherwise we would take $n \in\{[b]+1,[b]+2, \ldots\}$ ). Also if there exists a $\sigma, 0 \leq \sigma<\infty$ with

$$
\sup _{t \in[0, \infty)} \int_{a}^{b} \tau(s) k(t, s) d s=\int_{a}^{b} \tau(s) k(\sigma, s) d s
$$

then one could replace (2.64) with

$$
R<w(M R) \sup _{t \in[0, \infty)} \int_{a}^{b} \tau(s) k(t, s) d s
$$

Proof. Here let $E, \mathbf{E}^{k}, E^{k}, F_{n}$, and $F$ be as in Theorem 2.18. Let

$$
U=\left\{y \in E:|y|_{n}<r \forall n \in N\right\}, \quad V=\left\{y \in E:|y|_{n}<R \forall n \in N\right\},
$$

and note that for each $n \in N$ that

$$
U_{n}=\left\{y \in E_{n}:|y|_{n}<r\right\}, \quad V_{n}=\left\{y \in E_{n}:|y|_{n}<R\right\} .
$$

Also let

$$
C=\left\{y \in E: y(t) \geq 0 \text { on }[0, \infty), y(t) \geq M|y|_{n^{\prime}} \forall t \in[a, b], \forall n \in N\right\},
$$


and note that for each $n \in N$ that

$$
\overline{C_{n}}=C_{n}=\left\{y \in E_{n}: y(t) \geq 0 \text { on }[0, n], y(t) \geq M|y|_{n^{\prime}}, \forall t \in[a, b]\right\} .
$$

Finally we could take $Y=B(0, R+1)$. As in Theorem 2.18 clearly (2.36) and (2.37) hold; we need only to note that if $y \in \overline{W_{n}}$ then from (2.62) and (2.63) one has

$$
\begin{gathered}
\left|F_{n} y(t)\right| \leq \int_{0}^{n} g(s) f(s, y(s)) d s \quad \text { for } t \in[0, n], \\
F_{n} y(t) \geq M \int_{0}^{n} g(s) f(s, y(s)) d s \quad \text { for } t \in[a, b],
\end{gathered}
$$

so

$$
F_{n} y(t) \geq M\left|F_{n} y\right|_{n} \quad \text { for } t \in[a, b]
$$

Next we show that (2.39) is satisfied. Let $y \in \partial \Omega_{n}=\partial U_{n} \cap \overline{C_{n}}$. Then $|y|_{n}=r$ and this together with (2.22) yields

$$
\left|F_{n} y(t)\right| \leq \psi\left(|y|_{n}\right) \int_{0}^{n} k(t, s) \phi(s) d s \leq \psi(r) \sup _{t \in[0, \infty)} \int_{0}^{\infty} k(t, s) \phi(s) d s
$$

for $t \in[0, n]$, and so (2.23) yields

$$
\left|F_{n} y\right|_{n} \leq \psi(r) \sup _{t \in[0, \infty)} \int_{0}^{\infty} k(t, s) \phi(s) d s<r=|y|_{n} .
$$

Now let $y \in \partial W_{n}=\partial V_{n} \cap \overline{C_{n}}$. Then $|y|_{n}=R$ and $y(t) \geq M|y|_{n}=M R$ for $t \in[a, b]$ (in particular $y(t) \in[M R, R]$ for $t \in[a, b])$. Now (2.64) implies

$$
\begin{aligned}
\left|F_{n} y(t)\right| & =\int_{0}^{n} k(t, s) f(s, y(s)) d s \\
& \geq \int_{a}^{b} k(t, s) f(s, y(s)) d s \\
& \geq \psi(M R) \int_{a}^{b} k(t, s) \tau(s) d s,
\end{aligned}
$$


so (2.65) yields

$$
\begin{aligned}
\left|F_{n} y\right|_{n} & \geq \psi(M R) \sup _{t \in[0, n]} \int_{a}^{b} k(t, s) \tau(s) d s \\
& \geq \psi(M R) \sup _{t \in[0,1]} \int_{a}^{b} k(t, s) \tau(s) d s \\
& >R=|y|_{n} .
\end{aligned}
$$

Thus (2.39) holds. Now essentially the same argument as in Theorem 2.18 guarantees that (2.41) and (2.42) hold.

Notice (2.40) is satisfied with $r=M r$. To see this fix $k \in N$ and take a subsequence $A \subseteq\{k, k+1, \ldots\}$ and let $x \in \overline{C_{n}}$ be such that $x \in \overline{W_{n}} \backslash \Omega_{n}$ (i.e., $R \geq|x|_{n} \geq r$ ) for some $n \in A$. Then $\min _{t \in[a, b]} x(t) \geq M|x|_{n} \geq M r=\gamma$, so as a result $\left|j_{k} \mu_{k, n} j_{n}^{-1} x\right|_{k}=\max _{t \in[0, k]}|x(t)| \geq$ $\gamma$. The result now follows from Theorem 2.20.

\section{References}

[1] R. P. Agarwal, J. H. Dshalalow, and D. O’Regan, “Fixed point theory for Mönch-type maps defined on closed subsets of Fréchet spaces: the projective limit approach," International Journal of Mathematics and Mathematical Sciences, no. 17, pp. 2775-2782, 2005.

[2] R. P. Agarwal, M. Frigon, and D. O'Regan, "A survey of recent fixed point theory in Fréchet spaces," in Nonlinear Analysis and Applications: to V. Lakshmikantham on His 80th Birthday. Vol. 1, 2, pp. 75-88, Kluwer Academic Publishers, Dordrecht, The Netherlands, 2003.

[3] R. P. Agarwal and D. O'Regan, "A Lefschetz fixed point theorem for admissible maps in Fréchet spaces," Dynamic Systems and Applications, vol. 16, no. 1, pp. 1-12, 2007.

[4] L. Gorniewicz, Topological Fixed Point Theory of Multivalued Mappings, vol. 495 of Mathematics and Its Applications, Kluwer Academic Publishers, Dordrecht, The Netherlands, 1999.

[5] A. Granas and J. Dugundji, Fixed Point Theory, Springer Monographs in Mathematics, Springer, New York, NY, USA, 2003.

[6] D. O'Regan, "An essential map approach for multimaps defined on closed subsets of Fréchet spaces," Applicable Analysis, vol. 85, no. 5, pp. 503-513, 2006.

[7] R. P. Agarwal, M. Meehan, and D. O'Regan, Fixed Point Theory and Applications, vol. 141 of Cambridge Tracts in Mathematics, Cambridge University Press, Cambridge, UK, 2001.

[8] W. V. Petryshyn, "Existence of fixed points of positive $k$-set-contractive maps as consequences of suitable boundary conditions," Journal of the London Mathematical Society, vol. 38, no. 3, pp. 503-512, 1988.

[9] S. Park, "A unified fixed point theory of multimaps on topological vector spaces," Journal of the Korean Mathematical Society, vol. 35, no. 4, pp. 803-829, 1998.

[10] R. P. Agarwal and D. O’Regan, "Homotopy and Leray-Schauder principles for multi maps," Nonlinear Analysis Forum, vol. 7, no. 1, pp. 103-111, 2002.

[11] L. Górniewicz and A. Granas, "Some general theorems in coincidence theory. I," Journal de Mathématiques Pures et Appliquées, vol. 60, no. 4, pp. 361-373, 1981.

[12] D. O’Regan, "A Krasnoselskii cone compression theorem for $\mathfrak{U}_{c}^{\kappa}$ maps," Mathematical Proceedings of the Royal Irish Academy, vol. 103A, no. 1, pp. 55-59, 2003.

[13] M. Izydorek and Z. Kucharski, "The Krasnosielski theorem for permissible multivalued maps," Bulletin of the Polish Academy of Sciences. Mathematics, vol. 37, no. 1-6, pp. 145-149, 1989.

[14] L. V. Kantorovich and G. P. Akilov, Functional Analysis in Normed Spaces, The Macmillan, New York, NY, USA, 1964.

[15] D. O'Regan, "Leray-Schauder and Krasnoselskii fixed point theory in Fréchet spaces for Urysohn type operators,", in Legacy of the Legend, V. Lakshmikantham, Cambridge Scientific. 
[16] R. P. Agarwal and D. O'Regan, "Multivalued nonlinear equations on the half line: a fixed point approach," The Korean Journal of Computational \& Applied Mathematics, vol. 9, no. 2, pp. 509-524, 2002.

[17] R. P. Agarwal and D. O'Regan, "Lefschetz fixed point theorems for compact morphisms," Fixed Point Theory, vol. 7, no. 2, pp. 175-190, 2006.

[18] Z. Dzedzej, "Fixed point index theory for a class of nonacyclic multivalued maps," Dissertationes Mathematicae, vol. 253, pp. 1-58, 1985. 